\title{
Stage-Specific Differential Gene Expression of Glutathione Peroxidase in Leishmania Major and Leishmania Tropica
}

\author{
Nasrin Amiri-Dashatan ${ }^{1}$, Mehdi Koushki², \\ Mostafa Rezaei-Tavirani ${ }^{1}$, Nayebali Ahmadi*1,3
}

\begin{abstract}
Background: Leishmania $(L)$ major and L. tropica are the etiological agents of cutaneous leishmaniosis. Leishmania species cause a board spectrum of phenotypes. A small number of genes are differentially expressed between them that have likely an important role in the disease phenotype. Procyclic and metacyclic are two morphological promastigote forms of Leishmania that express different genes. The glutathione peroxidase is an important antioxidant enzyme that essential in parasite protection against oxidative stress and parasite survival. This study aimed to compare glutathione peroxidase (TDPX) gene expression in procyclic and metacyclic and also interspecies in Iranian isolates of $L$. major and $L$. tropica.

Methods: The samples were cultured in Novy-Nicolle-Mc Neal medium to obtain the promastigotes and identified using PCR-RFLP technique. They were then grown in RPMI1640 media for mass cultivation. The expression level of TDPX gene was compared by Real-time PCR.

Results: By comparison of expression level, up-regulation of TDPX gene was observed (5.37 and 2.29 folds) in L. major and L. tropica metacyclic compared to their procyclic, respectively. Moreover, there was no significant difference between procyclic forms of isolates, while 3.05 folds up-regulation in metacyclic was detected in L. major compared L. tropica.

Conclusions: Our data provide a foundation for identifying infectivity and high survival related factors in the Leishmania spp. In addition, the results improve our understanding of the molecular basis of metacyclogenesis and development of new potential targets to control or treatment and also, to the identification of speciesspecific factors contributing to virulence and pathogenicity in the host cells.
\end{abstract}

Keywords: Glutathione peroxidase, Leishmania, L. major, L. tropica, Quantitative Real-time PCR.

\section{Introduction}

Leishmaniasis is a global parasitic disease caused by various species of Leishmania (L) that refer to the variety of diseases including self-healing cutaneous leishmaniasis (CL) to fatal visceral leishmaniasis (VL) (1). CL is the most common form of leishmaniasis and highly regarded as a significant public health problem in many tropical and subtropical regions $(2,3)$. In the old world including Iran, anthroponotic (ACL) and zoonotic cutaneous leishmaniasis (ZCL) are two main forms of CL which are caused by $L$. tropica, $L$. major, respectively (2).

The emergence of leishmaniasis is not only associated with host immunity condition but also the type of parasite species is effective (4). The life cycle of Leishmania genus replacement between extracellular promastigotes and intracellular amastigotes. Promastigote forms also

1: Proteomics Research Center, Faculty of Paramedical Sciences, Shahid Beheshti University of Medical Sciences, Tehran, Iran.

2: Department of Clinical Biochemistry, School of Medicine, Zanjan University of Medical Sciences, Zanjan, Iran.

3: Department of Medical Lab Technology, Faculty of Paramedical Sciences, Shahid Beheshti University of Medical Sciences, Tehran, Iran.

*Corresponding author: Nayebali Ahmadi; Tel: +98 22714248; E-mail: nayebalia@sbmu.ac.ir.

Received: 3 Jul, 2020; Accepted: 19 Sep, 2020 
including two developmental stages including procyclic promastigotes and metacyclic promastigotes. Switch from non-infective procyclic (log phase) into the infective metacyclic (stationary phase) is accompanied by several morphological and genome expression variation (5). The alteration of the gene expression levels of the metacyclic leads to the emergence of parasitic infectious potency as well as adaptation to the mammalian host cell environment (6). Comparative analysis of gene expression level between both $L$. tropica, L. major and their metacyclogenesis process may serve to improve our understanding of molecular differences between these two species and also their metacyclogenesis stages in distinct species (6). Recent studies have also suggested the involvement of reactive oxygen species (ROS) in the pathogenesis of various parasitic diseases $(7,8)$. The ROS overproduction can lead to an imbalance between oxidants and antioxidants at cellular or systemic levels, causing the oxidative stress. Increased antioxidant activity may involve in pathogenicity and survival rate inside host cells. The ROS as endogenous species are produced in the mitochondrial respiratory chain during the normal metabolic process $(7,8)$. Antioxidants have the central role to neutralize ROS including the expression level of genes encoding glutathione peroxidase (TDPX) and tryparedoxin peroxidase (TRYP1) $(6,7)$. Once there is the involvement of oxidative stress in physiological and pathological processes, it is necessary to know the role of antioxidant enzymes such as TDPX between different stages and species of Leishmania. Glutathione peroxidase comprises an enzyme group that acts as a vital antioxidant by reducing hydro-peroxides. Furthermore, one of the vital processes for the survival of the Leishmania parasite in different environments is the detoxification of peroxides. This is the first study that describes the expression level of a gene encoding glutathione peroxidase protein (Uniprot ID: Q4Q9B4, Gene ID: 5652808) during the metacyclogenesis process of Iranian isolates of $L$. tropica and L. major. Figure 1 shows a schematic picture of this study workflow. The results of this work could inform about a potential drug target for leishmaniasis control or its treatment. Thus, the aim of this study was to evaluate the TDPX gene expression level in procyclic and metacyclic promastigotes and also interspecies in Iranian isolates $L$. major and L. tropica by quantitative Real-time PCR technique.

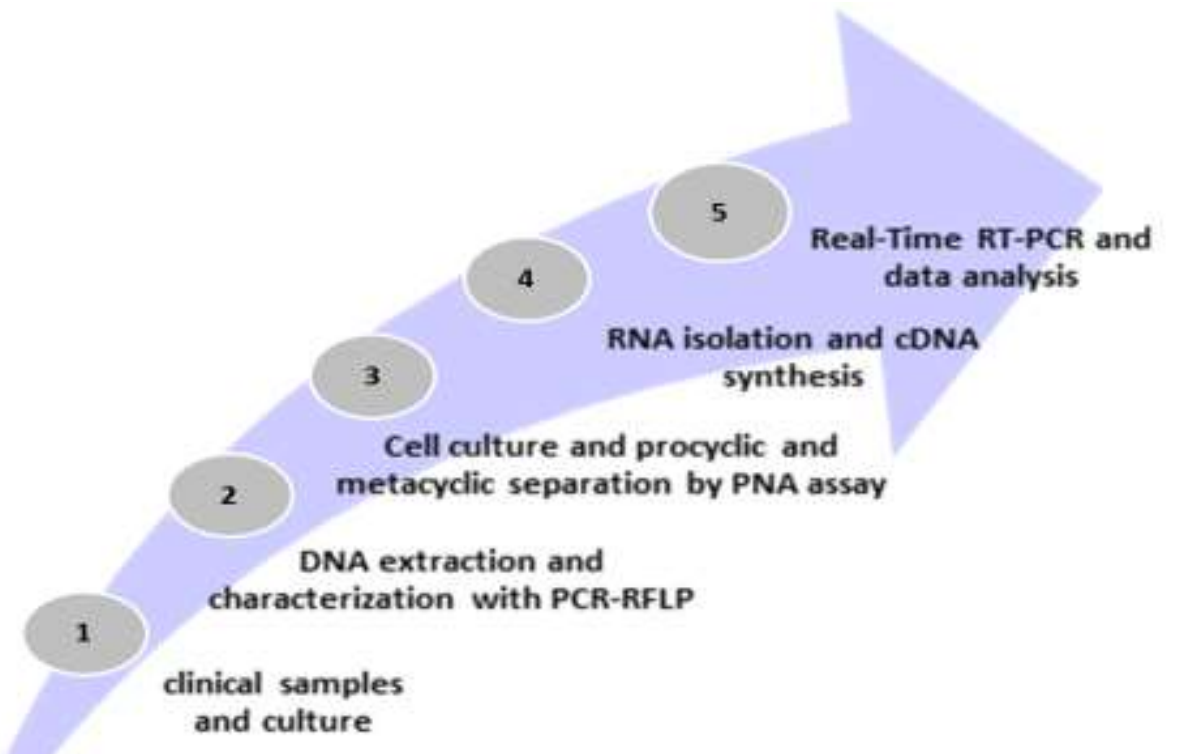

Fig. 1. Experimental workflow of quantitative Real-time PCR analysis of glutathione gene expression level in L. major and $L$. tropica isolates. 


\section{Materials and methods}

\section{Samples culture and characterization}

In this study, we used the Leishmania parasite isolates obtained from the cutaneous lesions of Iranian patients infected with $L$. major and $L$. tropica from an endemic place for each species. Written informed consent was obtained from each patient prior to participation. All the subjects were new cases, without taking medication. The primary isolates initially were grown on N.N.N (Novy-Nicolle-Mc Neal) medium and for mass production, parasites were cultured in RPMI-1640 medium (Gibco, Germany) supplemented with 10-15\% fetal bovine serum (Gibco, Germany) and $100 \mathrm{U} / \mathrm{ml}$ penicillin and $100 \mu \mathrm{g} / \mathrm{ml}$ streptomycin and incubated at $24-25^{\circ} \mathrm{C}$. In order to sample characterization, parasites DNA was extracted from promastigotes using the DNGPLUS commercial kit (SinaClon-Iran) according to the manufacturer's instructions. We performed a polymerase chain reaction-restriction fragment length polymorphism (PCR-RFLP) methods with internal transcribed spacer1 (ITS1) according to the method described previously (9). Since in this study procyclic and metacyclic promastigotes were evaluated for glutathione peroxidase expression level, peanut agglutinin (PNA; Sigma) assay and morphometric analysis were used to separate of purified procyclic and metacyclic stages as described previously $(5,9)$.

\section{RNA extraction and cDNA synthesis}

Total RNA was extracted from $10^{7}$ promastigotes (procyclic and metacyclic) of $L$. major and $L$. tropica samples during the log-phase using RiboEx reagent (GeneAll Biotech, Korea) according to the manufacturer's protocol. The quality and quantity of the RNA were evaluated by electrophoresis and Nanodrop 1000, respectively. To prevention of any gene contamination, total RNA was subjected to DNase I enzyme (Fermentas, Burlington, Canada). Three independent RNA samples were used for each real-time PCR experiment. Complementary DNA (cDNA) was synthesized using $3 \mu \mathrm{g}$ RNA, $1 \lambda$ oligo-dT (Thermofisher,
USA) using a RevertAid ${ }^{\mathrm{TM}}$ First Strand cDNA Synthesis Kit (Thermo Fisher Scientific). The gene expression analysis was assessed by qRTPCR using SYBR Green 2x Master Mix Green (Ampliqon). RT-qPCR was performed by a StepOnePlus ${ }^{\mathrm{TM}}$ Real-Time PCR System (Applied Biosystems, Foster City, CA).

\section{Quantitative Real-time PCR method}

Real-time PCR was conducted to evaluate the differences in the RNA expression level of TDPX gene among $\log$ (procyclic) and stationary (metacyclic) phases of $L$. major and L. tropica samples. Specific primers were designed by Gene Runner software version 6.5 .50 (wwwgenerunner.net). The sequence of the forward $(\mathrm{F})$ and reverse $(\mathrm{R})$ primers used in RTqPCR are the following; F: TTCATGGTACTGGCGTTCCC and R: ACCCTTGCACGTGTTCTTCA. PCR was performed in $20 \mu \mathrm{l}$ reactions containing $1 \mu \mathrm{l}$ cDNA target, $100 \mathrm{nM}$ forward and reverse primers and 1x SYBR Green RealQ Plus Master Mix (Ampliqon, DK-5230 Odense M, Denmark). Experiments were carried out in duplicate for target genes and Glyceraldehyde 3-phosphate dehydrogenase (GAPDH) gene as a housekeeping gene using a StepOne TM Real -time PCR System (Applied Biosystems, Life Technologies, USA). The PCR condition was as follows: activation at $95{ }^{\circ} \mathrm{C}$ for $3 \mathrm{~min}$, amplification at 95 ${ }^{\circ} \mathrm{C}$ for $10 \mathrm{~s}, 60^{\circ} \mathrm{C}$ for $1 \mathrm{~min}$ for 40 cycles. The data were normalized against the GAPDH transcript level and $2^{-\Delta c c t}$ method was used to calculate the relative expression.

\section{Statistical Analysis}

The data are reported as the mean \pm standard deviations (SDs). The significance of differences for expression levels in procyclic and metacyclic stages of $L$. major and L. tropica isolates was determined by Student's $t$-test and the level of significance acceptable was 95\% ( $p<0.05$ ). Statistical analyses were performed with GraphPad Prism 5.0 (GraphPad Software, Inc., San Diego, CA). 


\section{Results}

The identities of the isolates were performed by using PCR-RFLP technique (6). Real-time RTPCR was used to evaluate the relative expression level of TDPX gene in the developmentally procyclic and metacyclic stages of $L$. major and $L$. tropica Iranian isolates and to compare it with each other. Fig. 2a shows a significant upregulation of the TDPX gene in the metacyclic stage of $L$. major isolates (5.37 folds; $\mathrm{p}<0.001$ ) compared to its procyclic stage. Also, this gene in the metacyclic stage of $L$. tropica isolates significantly upregulated (2.29 folds; $\mathrm{p}<0.01)$

a)

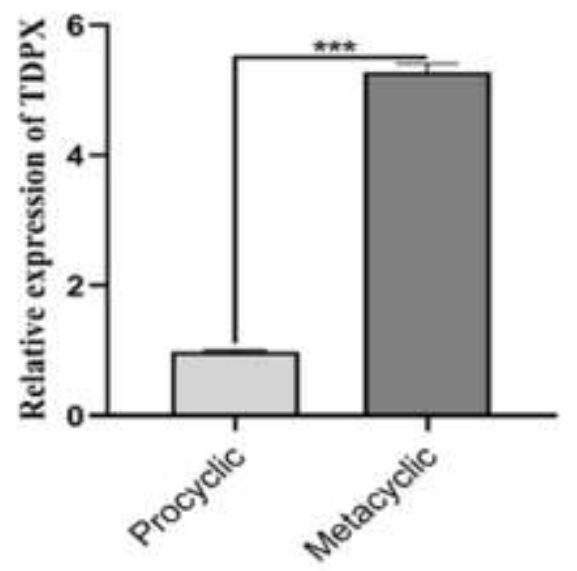

L. major compared with the procyclic stage (Fig.2b). In addition, the relative expression level of the TDPX gene between each specific stage of the $L$. major and L. tropica isolates was investigated, and no significant differences were detected between procyclic stages of two studied species (Fig. 3a), while TDPX gene expression level difference was significant between their metacyclic stages. According to Fig.3b TDPX gene was expressed 3.05 folds $(\mathrm{p}<0.01)$ in $L$. major metacyclic compared to $L$. tropica metacyclic.

b)

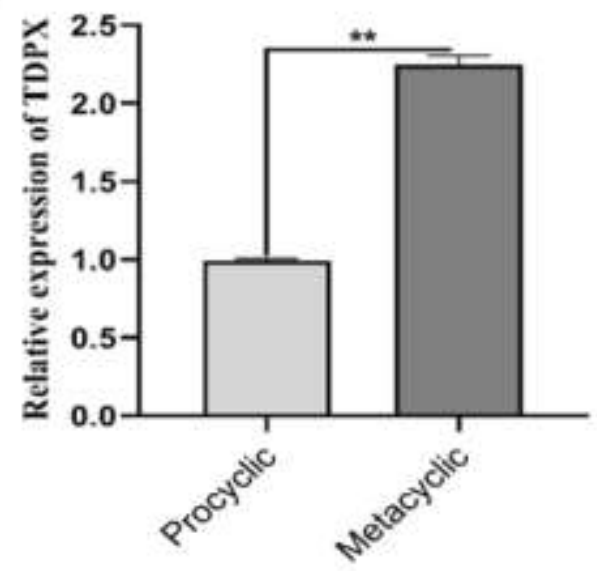

L. tropica

Fig. 2. Comparison of TDPX gene expression level in procyclic and metacyclic promastigotes forms of a) $L$. major and b) $L$. tropica Iranian isolates. $* * \mathrm{p}<0.01 ;$ and $* * * \mathrm{p}<0.001$.

a)

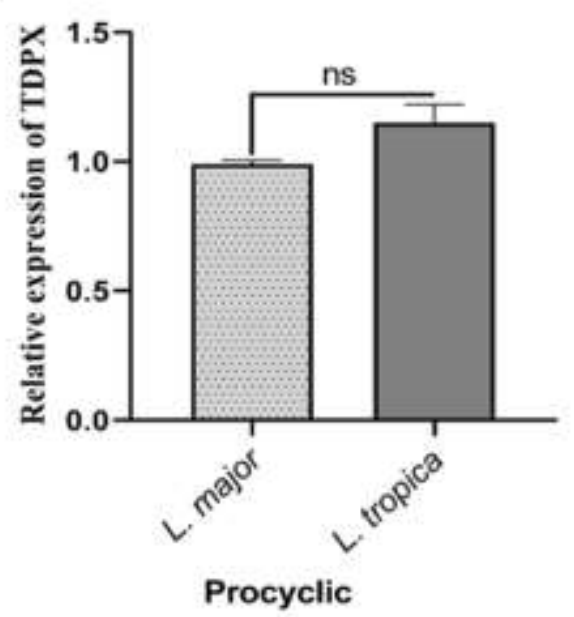

b)

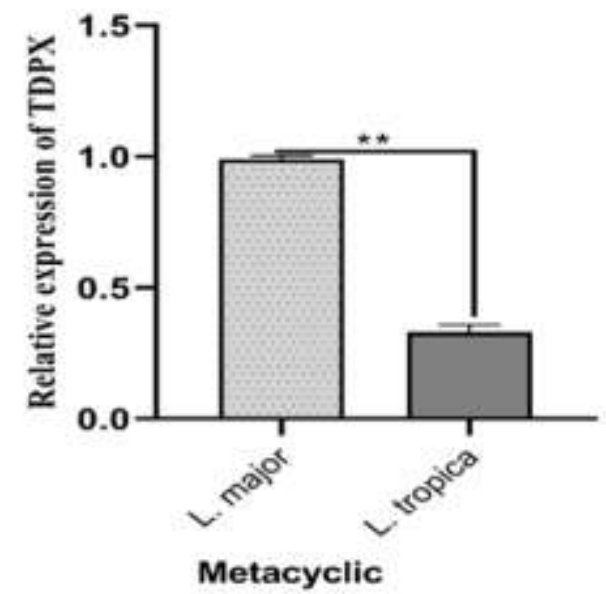

Fig. 3. Comparison of TDPX gene expression level in a) procyclic and b) metacyclic promastigotes forms of Iranian isolates of $L$. major and L. tropica. $* * \mathrm{p}<0.01$. 


\section{Discussion}

Leishmania parasites pass through different developmental phases including procyclic promastigote, metacyclic promastigote and amastigote forms in mammalian host cells with an alteration in gene expression profile and morphological changes. The parasites adapt themselves to changes in the infective stage and environment condition, in order to survive inside host cells. The full molecular basis of the procyclic to metacyclic conversion is limited. Large scale methods such as microarray genome analysis enable the studies of the gene expression level differences between life stages of the parasite and several studies exist in this field $(6,10-13)$. Studies have also been reported by real-time PCR to investigate on parasite gene expression $(6,14)$. We, therefore, chose to study the alteration of TDPX gene expression in Iranian isolates of $L$. major and $L$. tropica during metacyclogenesis, and also between species, that may contribute to the metacyclogenesis process and hence may play a role in Leishmania infectivity, pathogenicity, survival and ulcer phenotype differences among studied isolates.

TDPX protein is an antioxidant enzyme involved in response to oxidative stress that is a therapeutics opportunity (15) and the parasites need it to preadaptation and survive in the host intracellular environment. According to literature, antioxidant gene expression changes such as TDPX occurs in metacyclic compared with procyclic during the metacyclogenesis process. When procyclic promastigotes transition into metacyclic promastigotes forms, is accompanied by emerging of motility-related protein expression. Therefore, an increased level of proteins involved in motility is in agreement with high parasite movement and infectivity. The reactive oxygen species (ROS) as endogenous species are produced in the mitochondrial respiratory chain during the normal metabolic process (7, 9). Antioxidants have the central role to neutralize ROS including glutathione peroxidases (TDPX) and tryparedoxin peroxidases (TRYP1) (7). According to our data, the TDPX gene upregulated in the metacyclic stage of both $L$. major and L. tropica isolates. Overexpression of this protein as a part of the antioxidant enzyme class confirms the parasite answer to oxidative stress. Similarly, in our previous work on the protein profile of $L$. tropica during the metacyclogenesis process by using proteomics method, this protein upregulated in the metacyclic stage compared with procyclic stage in proteome level, in which validates this paper results (5). The results of other studies on L. donovani antioxidant enzymes also revealed more expression in drug-resistant parasites (16). Other documents reported that there is a link between tryparedoxin peroxidase with survival, virulence and drug response in $L$. donovani (17). Furthermore, one of the vital processes for the survival of Leishmania parasite in the cellular environment is the detoxification of peroxides. With regard to the contents mentioned about the function of antioxidant proteins, it can be concluded that increased expression of the TDPX gene in metacyclic forms of $L$. major and $L$. tropica isolates in this study, is because promastigotes undergo a high level of replication. It also naturally in the face of increased ROS during cell division due to high energy consumption and even age rising in procyclic and metacyclic forms increased the antioxidant and cell redox homeostasis related proteins. Since the metacyclic is an infective form of Leishmania and able to interact with the mammalian cells, alteration in genome expression during procyclic conversion into metacyclic form could be a way for pre-adaptation to host cell environment and intracellular survival. For this, the Leishmania alters some genes expression level such as TDPX to compatibility with the oxidative stress conditions of the host cells. Furthermore, overexpression of parasite protective complex could indicate the significant role of this gene in host-parasite interaction and increased infectivity of parasites. Similarly, previous studies using genomic microarrays and proteomics analysis showed more abundant expression of gene and protein in metacyclic and amastigote forms $(18,19)$. In the current study, a comparison of TDPX gene expression level between the procyclic of studied parasites showed no significant differences between L. major and L. tropica isolates, whereas the corresponding level significantly differed between L. major and L. tropica metacyclic forms. A few numbers of species-specific genes affected pathogenesis and clinical manifestation, but the expression level of genes usually differ between 
different species (20). Since ZCL phenotype is severe rather than ACL, the increased level of TDPX gene expression in L. major can be related to high adaptive and survival ability in host cells and emerging of intense ulcers caused by $L$. major. Therefore, this gene may be a potential proper target for metacyclogenesis and infectivity stop and improves new potential targets to control or treatment. However, further studies must be undertaken with a large number of isolates and detailed investigations to better understand the distinct gene expression pattern found between $L$. major and L. tropica.

It is the first study is evaluating on the TDPX gene in a number of different Iranian cutaneous Leishmania parasite species isolates ( $L$. major and $L$.

\section{References}

1. Assimina Z, Charilaos K, Fotoula B. Leishmaniasis: An overlooked public health concern. Health Sci J. 2008;2(4):196-205.

2. Ahmadi N, Modiri M, Mamdohi S. First survey of cutaneous leishmaniasis in Borujerd county, western Islamic Republic of Iran. East Mediterr Health J. 2013;19(10):847-853.

3. Postigo JAR. Leishmaniasis in the world health organization eastern mediterranean region. Int $\mathrm{J}$ Antimicrob Agents. 2010;36(Supp1):S62-65.

4. Livneh A, Youngster I, EI-On Y, Berkovitch M, Abu-Kishk I. The lack of effectiveness of hyperbaric oxygenation as a treatment for Leishmania major in a mouse model. Acta Parasitol. 2015;60(2):345-349. 5. Amiri-Dashatan N, Rezaei-Tavirani M, Ahmadi N. A quantitative proteomic and bioinformatics analysis of proteins in metacyclogenesis of Leishmania tropica. Acta Tropica. 2020;202:105227.

6. Amiri-Dashatan N, Koushki M, Ahmadi N. Comparison of gene expression of pyruvate kinase and tryparedoxin peroxidase in metacyclic promastigote forms of Leishmania (L.) tropica and L. major by real-time PCR. Ann Parasitol. 2020;66(1):13-18.

7. He L, He T, Farrar S, Ji L, Ma X. Antioxidants maintain cellular redox homeostasis by elimination of reactive oxygen species. Cell Physiol Biochem. 2017;44(2):532-553. tropica) including ZCL and ACL forms. The average expression of the TDPX gene was different significantly between metacyclic and procyclic stages of isolates. In addition, TDPX gene expression was higher in $L$. major compared to $L$. tropica. Our findings suggest that TDPX can be considered as potential molecular markers for metacyclogenesis and screening of cutaneous leishmania species isolates.

\section{Acknowledgements}

This project was supported by the Proteomics Research Center of Shahid Beheshti University of Medical Science.

The authors declare that they have no conflicts of interest with the contents of this article.

8. Nathan C, Cunningham-Bussel A. Beyond oxidative stress: an immunologist's guide to reactive oxygen species. Nature Reviews Immunology. 2013;13:349-361.

9. Tabrizi F, Seyyed Tabaei S, Ahmadi N, Arefi Oskouie A. A nuclear magnetic resonance- based metabolomic study to identify metabolites differences between Iranian isolates of Leishmania major and Leishmania tropica. Iran J Med Sci. 2020;46:(2-10).

10. Saxena A, Lahav T, Holland N, Aggarwal G, Anupama A, Huang Y, et al. Analysis of the Leishmania donovani transcriptome reveals an ordered progression of transient and permanent changes in gene expression during differentiation. Mol Biochem Parasitol. 2007;152(1):53-65.

11. Akopyants NS, Matlib RS, Bukanova EN, Smeds MR, Brownstein BH, Stormo GD, et al. Expression profiling using random genomic DNA microarrays identifies differentially expressed genes associated with three major developmental stages of the protozoan parasite Leishmania major. Mol Biochem Parasitol. 2004;136(1):71-86.

12. Torres DC, Adaui V, Ribeiro-Alves M, Romero GAS, Arevalo J, Cupolillo E, et al. Targeted gene expression profiling in Leishmania braziliensis and Leishmania guyanensis parasites isolated from Brazilian patients with different antimonial treatment outcomes. Infect Genet Evol. 2010;10(6):727-733. 
13. Cohen-Freue G, Holzer TR, Forney JD, McMaster WR. Global gene expression in Leishmania. Int J Parasitol. 2007;37(10):1077-86.

14. Mohebali M,Kazemirad E, Hajjaran H, Kazemirad E, Oshaghi MA, Raoofian R, et al. Gene expression analysis of antimony resistance in Leishmania tropica using quantitative real-time PCR focused on genes involved in trypanothione metabolism and drug transport. Arch Dermatol Res. 2019;311(1):9-17.

15. Lubos E, Loscalzo J, Handy DE. Glutathione peroxidase-1 in health and disease: from molecular mechanisms to therapeutic opportunities. Antioxidants \& redox signaling. 2011;15(7):1957-1997.

16. Andrade JM, Murta SMF. Functional analysis of cytosolic tryparedoxin peroxidase in antimonyresistant and -susceptible Leishmania braziliensis and Leishmania infantum lines. Parasit \& Vectors. 2014;7(406):1-9.
17. Iyer JP, Kaprakkaden A, Choudhary ML, Shaha C. Crucial role of cytosolic tryparedoxin peroxidase in Leishmania donovani survival, drug response and virulence. Mol Microbiol. 2008;68(2):372-91. 18. Rochette A, Raymond F, Ubeda JM, Smith M, Messier N, Boisvert S, et al. Genome-wide gene expression profiling analysis of Leishmania major and Leishmania infantum developmental stages reveals substantial differences between the two species. BMC genomics. 2008;9(255):1-26.

19. Moreira W, Legare D, Racine G, Roy G, OuelletteM. Proteomic analysis of metacyclogenesis in Leishmania infantum wild-type and PTR1 null mutant. EuPA Open Proteomics. 2014;4:171-183.

20. Peacock CS, Seeger K, Harris D, Murphy L, Ruiz JC, Quail MA, et al. Comparative genomic analysis of three Leishmania species that cause diverse human disease. nature genetics. 2007;39:839-847. 